Anne Aimable $^{1}$
Nathalie Jongen $^{1}$
Andrea Testino $^{1}$
Marcel Donnet $^{1}$
Jacques Lemaître $^{1}$
Heinrich Hofmann ${ }^{1}$
Paul Bowen

${ }^{1}$ Ecole Polytechnique Fédérale
de Lausanne (EPFL), Swiss
Federal Institute of
Technology, Materials Institute,
Powder Technology Laboratory
(LTP), Lausanne, Switzerland.

\author{
Research Article
}

\section{Precipitation of Nanosized and Nanostructured Powders: Process Intensification and Scale-Out Using a Segmented Flow Tubular Reactor (SFTR)}

\begin{abstract}
The successful scale-out and process intensification using a segmented flow tubular reactor (SFTR) for ultrafine $\mathrm{CaCO}_{3}, \mathrm{BaTiO}_{3}$, and nanosized $\mathrm{ZnO}$ from optimized minibatch $(20 \mathrm{~mL})$ conditions is presented. The capacity of the SFTR in process intensification was demonstrated by producing $\sim 5 \mathrm{~kg}$ batches of $\mathrm{BaTiO}_{3}$ powders with excellent batch-to-batch reproducibility. The SFTR scale-out or numbering-up capacity was demonstrated for a nanostructured $\mathrm{CaCO}_{3}$ in $500 \mathrm{~g}$ batches by scaling-out from one to six segmented flow tubular reactors run in parallel (scale-out/-up ratio of 5000 compared to lab batch experiments). The SFTR was then used to demonstrate its potential for nanosized $\mathrm{ZnO}$ powders producing $50 \mathrm{~g}$ lots of these nanopowders in a continuous process, a scale-out/up ratio of 250 compared to lab batch experiments without any loss of powder quality. The SFTR allows a precise control of precipitation conditions, leading to an excellent reproducibility in powder characteristics, and shows great promise as a simple production process of powders and advanced nanomaterials with highly controlled properties.
\end{abstract}

Keywords: Nanoparticles, Plug flow, Powders, Precipitation, Segmented flow tubular reactor Received: July 30, 2010; revised: August 26, 2010; accepted: October 19, 2010

DOI: $10.1002 /$ ceat.201000324

\section{Introduction}

Nanomaterials and nanotechnology attract an extraordinary amount of interest, as shown by the increasing amount of publications and patents published on the subject every year and the continuous growth of funding: in the United States, the federal funding for nanotechnology has increased from approximately $\$ 464$ million in 2001 to nearly $\$ 1.5$ billion in 2009 and was more than $\$ 5$ billion worldwide [1]. The unique properties of these nanomaterials bring them into a key position for a large spectrum of devices and systems [2]. But after 20 years of exciting new discoveries, researchers face the real challenge: to convert science-based findings and research laboratory methods into applied technologies and processes suitable for use in a commercial environment. This is more and

Correspondence: Dr. A. Aimable (anne.aimable@epfl.ch), Ecole Polytechnique Fédérale de Lausanne (EPFL), Swiss Federal Institute of Technology, Materials Institute, Powder Technology Laboratory (LTP), Station 12, 1015 Lausanne, Switzerland. more emphasized in current reviews on nanotechnology [3, 4], as, e.g., by Ashby et al. [5]: "Making effective design application use of new scientific findings that seemingly appear every day within the nanomaterial field is not as easy as might first appear", or by Pitkethly [6]: "There are still many challenges for nanomaterials companies to overcome before the potential is fully realized. These include: how to produce materials in volume commercially at viable prices...//...consistency and reliability in volume production."

Projects funded by the European Union also show this necessity in developing new solutions for nanopowder production in a volume that could lead to real applications. A specific call NMP-2008-2.1-2 Processing and Upscaling of Nanostructured Materials was dedicated to this issue in the seventh Research Program FP7 in 2008 Nanosciences, Nanotechnologies, Materials and New Production Technologies.

Over recent years a new type of tubular plug flow reactor the segmented flow tubular reactor (SFTR) - has been conceived and used in powder synthesis [7]. This type of tubular or segmented flow reactors has shown excellent performances in liquid-liquid reactions as microreactors with segmented flow in capillaries or microchannels [8-10]. The microfluidics 
of such reactors has been studied in detail $[11,12]$ showing the advantages of the increased mass and heat transfer between the two liquid phases, which enhance conversion and selectivity for phase transfer-catalyzed reactions [13]. These microreactors have also improved performance efficiency in liquid-liquid extraction [14] and have great potential in synthetic biology [15]. When compared with batch reactors, they are of great interest for both liquid-liquid reactions and powder production targets because they can be operated in a continuous mode and ensure controlled hydrodynamics, residence times, and reaction conditions.

For powder synthesis the main tool used has been the SFTR which has proved its versatility and its robustness through the preparation of different products: $\mathrm{CaCO}_{3}$ [16], $\mathrm{BaTiO}_{3}$ [17], copper, calcium and nickel-manganese oxalates $[18,19]$ with a high powder quality (chemical and phase composition, particle size, and shape) and reproducibility [20].

The use of droplet-based microfluidics proved to be a viable method for nanosized powders [21] but has not been fully exploited with respect to the scale-out/-up and process intensification potential which reactors such as the SFTR possess. This paper presents examples of both scale-out from a singletube to a six-tube reactor for nanostructured $\mathrm{CaCO}_{3}$ and the process intensification showing its capabilities to produce $\mathrm{kg}$ quantities of high-quality $\mathrm{BaTiO}_{3}$ powders. The main subject will focus on the synthesis of nonagglomerated redispersible nanoparticles of $\mathrm{ZnO}(<100 \mathrm{~nm})$ by using the SFTR.

$\mathrm{ZnO}$ has already many applications, such as gas sensors or varistors [22]. Optical properties are also widely exploited as a dye (zinc white) or UV-light emitter [23]. By decreasing the size, $\mathrm{ZnO}$ can be used as quantum dots [24]. The synthesis of $\mathrm{ZnO}$ nanoparticles $(<100 \mathrm{~nm})$ with a homogeneous morphology and a narrow particle size distribution was investigated in a previous study using a small batch reactor (reactor volume $40 \mathrm{~cm}^{3}$, powder yield $0.2 \mathrm{~g}$ ) [25]. The size and morphology of the precipitated $\mathrm{ZnO}$ can be tuned with the addition of polymers or by modifying the $\mathrm{pH}$. By conducting the synthesis under basic conditions, the morphology was modified towards a flower-like shape with an aspect ratio of the primary particles increasing with the $\mathrm{pH}$. Under slightly acidic conditions, the anionic additives poly(acrylic acid) (PAA 2000, Dispex A40) and sodium dodecyl sulfate (SDS) reduced the final particle size and degree of agglomeration by an assumed preferential adsorption on the (0001) positive surface. Nonagglomerated equiaxed nanoparticles (around $100 \mathrm{~nm}$ ) with a narrow size distribution ( $\operatorname{span}<1$ ) could be obtained with a very good reproducibility. These features make these materials very interesting for further applications in electronic devices and should allow a higher density after sintering.

In order to investigate more such properties and conduct ceramic processing and sintering tests, it is necessary to have batches of powders of minimum size, at least $50 \mathrm{~g}$, whereas a typical laboratory batch process only produced $0.2 \mathrm{~g}$ per batch. The present paper describes the transfer of $\mathrm{ZnO}$ synthesis from the minibatch to a continuous production using the SFTR with a significant scale-up ratio of 250 . The powder characteristics obtained with a simple batch volume or with the SFTR is compared and the robustness of the SFTR is investigated. The effect of the micromixer used in the process on the material properties is also studied, as it is well-known that the mixing conditions can determine the resulting product properties in precipitation systems, especially influencing the particle size [26-28]. A key factor for nanopowder production is its colloidal stability and capacity to be dried and redispersed without loss of powder quality. There are many nanopowders available in small quantities and some can be stabilized in suspension but suffer significant nonreversible agglomeration on drying. The cost of transporting and storing suspensions even at $30 \%$ weight solids fraction is significantly higher than transporting a dried powder - the key is the ability to redisperse the powder after drying. This will also be demonstrated for the $\mathrm{ZnO}$ powder investigated in the current study.

\section{Materials and Methods}

\subsection{The Segmented Flow Tubular Reactor: SFTR}

The SFTR has been developed to overcome the classical problems of powder production scale-up from batch processes, which are mainly linked with mixing, homogeneity, and heat transfer. A schematic view of the SFTR is given in Fig. 1a). The SFTR is composed of three distinct parts: a micromixer which ensures that the coreactants are efficiently mixed, a segmenter, and a tubular reactor, placed in a thermostatic bath. The reactants are introduced through the micromixer where the initial supersaturation is created. The reacting mixture is then segmented by an immiscible solvent: nitrogen gas in the case of $\mathrm{BaTiO}_{3}$ precipitation, dodecane in the case of $\mathrm{CaCO}_{3}$, and $\mathrm{ZnO}$ precipitation. Small suspension volumes $\left(\sim 0.2 \mathrm{~cm}^{3}\right)$ are thus created, producing microdroplets or microreactors, where precipitation and growth takes place. These small volumes ensure a high homogeneity inside each droplet, all circulating through the tube with an identical history (e.g., residence time and heat exchange). Finally, fouling at the wall surface is avoided due to the immiscible fluid by forming a thin film at the surface [12] and thus allowing long continuous process times $(>12 \mathrm{~h})$. The residence time is determined by the pump flow speeds, the tube length, and reaction kinetics and was around $10 \mathrm{~min}$ for $\mathrm{ZnO}$ (tube diameter: $4 \mathrm{~mm}$, tube length: $20 \mathrm{~m}$ ). This is a steady-state process in which each step, mixing and ageing, is well separated, leading to a better control and reproducibility. If needed, after the tubular reactor a heat-exchanger can be placed to cool down the reacting mixture to room temperature and stop the reaction. Then a separation unit or decanter allows the separation of the aqueous suspension and the immiscible fluid. The powder in the aqueous phase is collected, while dodecane is recycled in the process for environmental and cost considerations.

\subsection{Micromixers}

In precipitation systems, the role of the mixing is preponderant and can affect to a great extent the final material, its phase purity, morphology, size, and particle size distribution. Two micromixer designs were investigated in this study: The Y-configuration (Fig. 1b)) consists of two impinging sheets mixing 


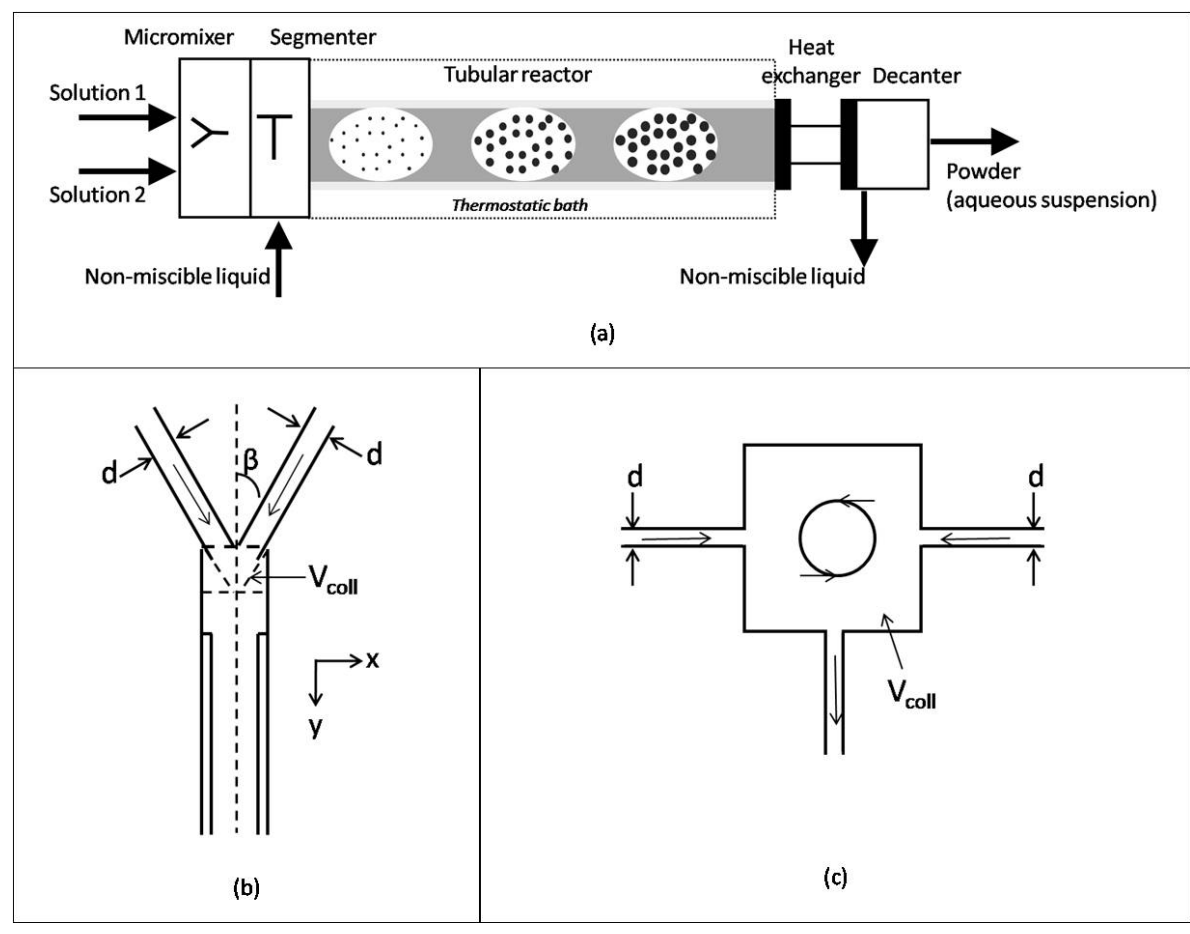

Figure 1. Schematic views: (a) SFTR, (b) Y-micromixer, (c) Roughton micromixer.

$D=\frac{k_{\mathrm{B}} T}{6 \pi \eta r}$

where $k_{\mathrm{B}}$ is the Boltzmann constant, $T$ is the temperature, $\eta$ is the dynamic viscosity of the precipitation medium, and $r$ is the ionic radius of the considered species. Taking these equations and the geometry into account, the Kolmogorov length and an approximate mixing time in the two micromixers were calculated $[28,31]$ and both geometries were used in the precipitation of $\mathrm{ZnO}$.

\subsection{Powder Characterization}

The phase identification of the precipitates was made with powder X-ray diffraction (XRD, Philips $\mathrm{X}$ 'Pert diffractometer, $\mathrm{Cu}-\mathrm{K}_{\mathrm{a}}$ radiation). XRD peak broadening was used to determine the size of the primary crystallite using the Scherrer equation (Eq. (5)). The instrumental broadening was determined

at the impingement zone $V_{\text {coll }}$ with an angle of $30^{\circ}$ which ensures an inelastic collision [29]; the Roughton configuration [30] is a T-mixer where the feed flows are displaced from a direct collision geometry to create an internal vortex in the mixing chamber $V_{\text {coll }}$ (Fig. 1c)). For these two designs, the micromixing efficiency can be approximated and compared by calculating the Kolmogorov length $[28,31]$. The specific energy dissipation rate $\varepsilon$ is determined by the geometry of the impingement zone and the speed of the fluid.

$\varepsilon=\frac{2 P}{m}$

Factor 2 results from the consideration of the two single sheets, which are assumed to be equal. $P$ is the power lost during the collision, which is simply the kinetic energy divided by the time in the direction of the $x$-axis, and $m$ is the mass over which $P$ is lost (mass of liquid in $V_{\text {coll }}$ ). The Kolmogorov length is given by Eq. (2):

$\lambda=\left(\frac{v^{3}}{\varepsilon}\right)^{1 / 4}$

where $\lambda$ is the Kolmogorov length, $v$ is the kinematic viscosity, and $\varepsilon$ is the energy dissipation rate.

One can then estimate a mixing time, defined as follows:

$t=\frac{(\lambda / 2)^{2}}{D}$

where $\lambda$ is the Kolmogorov length and $D$ is the species diffusivity. This last value can be calculated as: using alumina with a large crystal size $(>1 \mu \mathrm{m})$.

$d_{\mathrm{XRD}}=\frac{K \lambda_{\mathrm{X}}}{\beta_{\mathrm{Xp}} \cos (\theta)}$

where $K$ is equal to $0.9, \lambda_{\mathrm{X}}$ is the $\mathrm{X}$-ray wavelength, $\beta_{\mathrm{Xp}}$ is the integral breadth of the material, calculated using $\beta_{\mathrm{Xp}}=\sqrt{\left(\beta^{2}-\beta_{\text {alumina }}^{2}\right)}$, with the integral breadth $\beta$ defining the ratio of the area and height of the diffraction peak.

The powder morphologies were analyzed by scanning electron microscopy (SEM, Philips XL 30 FEG microscope). The SEM samples were prepared by dispersing the powder in ethanol. One drop of the suspension was then deposited on an aluminum support and dried in air. TEM micrographs were obtained from a Philips CM 200 TEM operating at $200 \mathrm{kV}$ and room temperature.

Brunauer-Emmett-Teller (BET)-specific surface areas $S_{\mathrm{BET}}$ $\left(\mathrm{m}^{2} \mathrm{~g}^{-1}\right)$ were estimated from $\mathrm{N}_{2}$ adsorption isotherms (Micromeritics Gemini 2375). The size of the primary particles, $d_{\mathrm{BET}}$ (nm) [32], was calculated by assuming spherical monodisperse particles (Eq. (6)), where $\rho$ is the density of the material.

$d_{\mathrm{BET}}=\frac{6000}{S_{\mathrm{BET}} \rho}$

Before BET measurements the samples were dried at $200^{\circ} \mathrm{C}$ in flowing nitrogen for $1 \mathrm{~h}$.

The particle size distribution (PSD) was collected using a centrifugal method (CPS, Disc Centrifuge Model DC 24000, or XDC, X-Ray Disc Centrifuge Brookhaven Instruments). For this measurement, powders were suspended in a $0.1 \mathrm{wt}-\%$ 
Table 1. Experimental data and geometric values used for the calculation of the micromixing times in the two micromixers for the $\mathrm{ZnO}$ precipitation system.

\begin{tabular}{llllll}
\hline Y-Micromixer & \multicolumn{5}{c}{ Roughton Micromixer } \\
\hline Internal diameter & $d[\mathrm{~m}]$ & $2 \cdot 10^{-3}$ & Internal diameter & $d[\mathrm{~m}]$ & $3 \cdot 10^{-4}$ \\
Flow rate & $Q\left[\mathrm{~m}^{3} \mathrm{~s}^{-1}\right]$ & $5 \cdot 10^{-6}$ & Flow rate & $Q\left[\mathrm{~m}^{3} \mathrm{~s}^{-1}\right]$ & $1.67 \cdot 10^{-7}$ \\
$\beta$ & angle $\left[^{\circ}\right]$ & 30 & Flow speed & $v\left[\mathrm{~m} \mathrm{~s}^{-1}\right]$ & 2.36 \\
Flow speed & $v_{\mathrm{x}}\left[\mathrm{m} \mathrm{s}^{-1}\right]$ & 0.79 & Volume of collision & $V_{\text {coll }}$ & $2 \cdot 10^{-9}$ \\
Dissipation rate & $\varepsilon\left[\mathrm{m}^{2} \mathrm{~s}^{-3}\right]$ & 109 & Dissipation rate & $\varepsilon\left[\mathrm{m}^{2} \mathrm{~s}^{-3}\right]$ & 466 \\
Kolmogorov length & $\lambda[\mu \mathrm{m}]$ & 9.8 & Kolmogorov length & $\lambda[\mu \mathrm{m}]$ & 6.8 \\
Mixing time & $t[\mathrm{~ms}]$ & 17 & Mixing time & $t[\mathrm{~ms}]$ & 10 \\
\hline
\end{tabular}

solution of PAA ( $\mathrm{pH} \sim 10$, concentration 0.01 to $0.25 \mathrm{wt}-\%$ ), and then treated $15 \mathrm{~min}$ with an ultrasonic horn. Median volume particle diameters $\left(d_{\mathrm{v} 50}\right)$ and either the distribution standard deviation or span $\left(d_{\mathrm{v} 90}-d_{\mathrm{v} 10}\right) / d_{\mathrm{v} 50}$ were used to characterize the widths of the distributions [32].

Thermogravimetric curves (TGA, Mettler TGA/DSC/TMA analyzer) were collected in the range from room temperature to $800^{\circ} \mathrm{C}$ under flowing air at a heating rate of $10^{\circ} \mathrm{C} \cdot \mathrm{min}^{-1}$.

\section{Barium Titanate Precipitation: Process Intensification}

Barium titanate precipitation was carried out using barium chloride and titanium tetrachloride in one reactant stream and sodium hydroxide in excess as the second stream. The experimental details and particle growth mechanism have been well described elsewhere where the reactant concentrations and temperatures were initially investigated in small-batch reactors $(50 \mathrm{~g})$ and then transferred to the SFTR $[25,33]$. The SFTR micromixer was of the Y-type. Nitrogen gas was used as a segmenting fluid and also protects against carbonate formation. The reaction was carried out at $95^{\circ} \mathrm{C}$ and the residence time in the reactor was $10 \mathrm{~min}$.

Regular ultrafine particles with an almost cubic morphology were obtained (see SEM picture in Fig. 2). The tetragonal phase was directly obtained and proved to be very pure with no traces of barium carbonate often found in even the best commercial powders. This powder presents a specific surface area of $S_{\mathrm{BET}}=10.5 \mathrm{~m}^{2} \mathrm{~g}^{-1}$. During early investigations small batches of up to $50 \mathrm{~g}$ were produced in short production runs ( 1 or $2 \mathrm{~h}$ ). To illustrate how the SFTR can intensify a process, increased batch sizes without loss of powder quality batches of 3 to $7 \mathrm{~kg}$ were produced by running the SFTR continuously for up to $30 \mathrm{~h}$. The ultrafine nature and high-quality powder is illustrated with a high degree of reproducibility, as shown by the comparison in PSDs measured on seven different synthesis batches (Fig. 3a) where the PSDs are almost indistinguishable. The $d_{\mathrm{v} 50}$ measured on seven different lots was $173 \mathrm{~nm}$, with a very low standard deviation of $1.7 \%$, illustrating the capacity of the SFTR in process intensification.

\section{Calcium Carbonate Precipitation: Scale-Up/-Out Demonstration}

Calcium carbonate precipitation has been studied intensively in our group for more than ten years $[16,20,34-37]$. The precipitation of calcite is

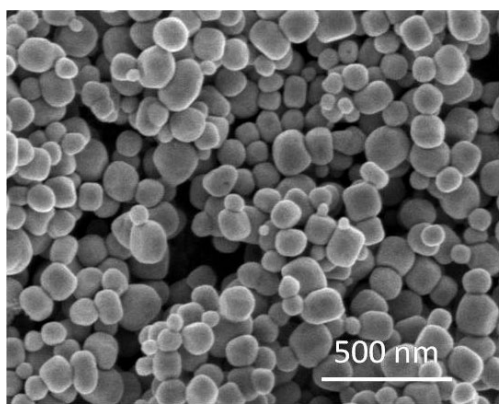

Figure 2. SEM picture of $\mathrm{BaTiO}_{3}$ ultrafine particles obtained using the SFTR.

controlled by the addition of seeds stabilized by PAA. These seeds are added to a potassium carbonate solution $\left(0.02 \mathrm{~mol} \mathrm{~L}^{-1}\right)$ as one stream, mixed with a calcium nitrate solution $\left(0.02 \mathrm{~mol} \mathrm{~L}^{-1}\right)$ as another stream, using a Y-type micromixer. The above route has been optimized with a $20 \mathrm{~mL}$ batch reactor [35] allowing the production of high-surface-area nanostructured powders. Dodecane was used as the segmenting fluid. The reaction was carried out at ambient temperature and pressure, and the residence time was $10 \mathrm{~min}$. To illustrate the stability of product quality as a function of reactor opera-

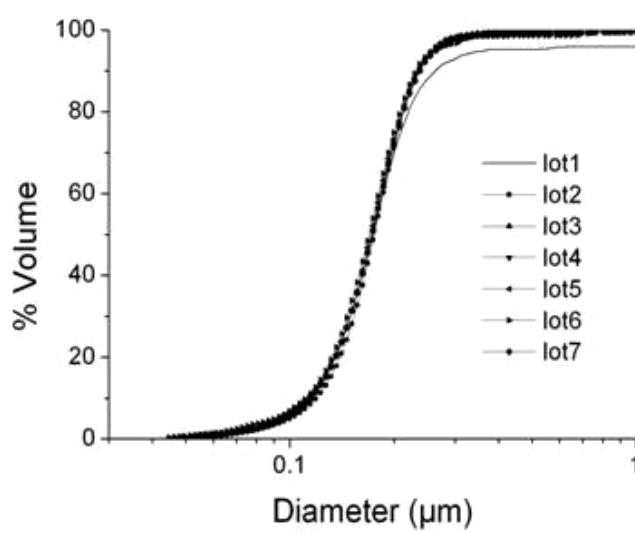

(a)

\begin{tabular}{cccc} 
& dv10 [nm] & dv50 [nm] & dv90 [nm] \\
\hline lot1 & 116 & 174 & 263 \\
lot2 & 119 & 175 & 235 \\
lot3 & 115 & 173 & 236 \\
lot4 & 116 & 178 & 245 \\
lot5 & 114 & 171 & 235 \\
lot6 & 111 & 169 & 231 \\
lot7 & 112 & 173 & 237 \\
\hline Average & $\mathbf{1 1 5}$ & 173 & 240 \\
STD(\%) & 2.3 & $\mathbf{1 . 7}$ & 4.5 \\
\hline
\end{tabular}

Figure 3. (a) Particle size distributions of seven different lots of $\mathrm{BaTiO}_{3}$ powders obtained using the SFTR, measured by XDC, (b) percentile volume diameters measured, average, and standard deviations. 
tion time, the SFTR was run continuously for $25 \mathrm{~h}$. To illustrate the scale-out concept, a multiple-tube configuration with six tubes running in parallel was carried out for a $15 \mathrm{~h}$ period, which needed $100 \mathrm{~L}$ of reactants.

Typical calcium carbonate particles are shown in Fig. 4. They have a slightly elongated shape but were pure calcite within the detection limits of X-ray powder diffraction [34]. The powders have SSAs around $35 \mathrm{~m}^{2} \mathrm{~g}^{-1}$, and the rough surface illustrates their nanostructured nature $\left(d_{\mathrm{XRD}}=67 \mathrm{~nm}\right)$. The PSDs for the minibatch, the single-tube SFTR as well as the multi-tube SFTR precipitates are shown in Fig. 5a) and are very similar. The span and $d_{\mathrm{v} 50}$ for each type of powder is shown in Fig. 5b). This scale-up of 5000 going from the minibatch reactor $\left(20 \mathrm{~cm}^{3}\right)$ to the $100 \mathrm{~L}$ of the multitube run without any loss of powder quality is remarkable and shows the power of the segmented flow reactor approach for the production and scale-out/-up of high-quality powders.

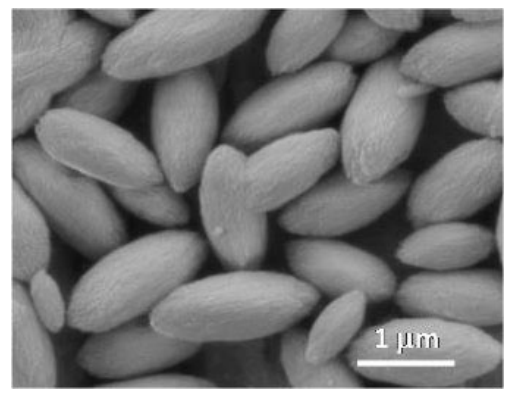

Figure 4. SEM picture of $\mathrm{CaCO}_{3}$ nanostructured particles obtained using the SFTR.

\section{$5 \quad$ Zinc Oxide Precipitation: Continuous Synthesis of Well-Dispersed Nanoparticles $<100 \mathrm{~nm}$}

\subsection{ZnO Precipitation Procedure}

In this study, the synthesis of $\mathrm{ZnO}$ using the continuous SFTR process is presented for the first time. The precipitation of

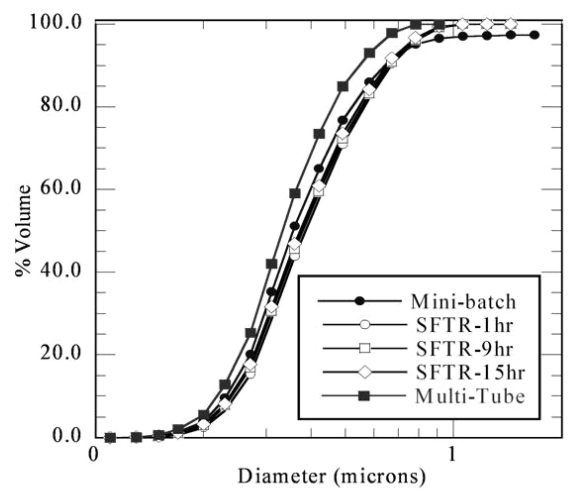

(a)

\begin{tabular}{ccc}
\hline & Span & $\mathrm{d}_{\mathrm{v} 50}[\mu \mathrm{m}]$ \\
\hline Mini-batch $(20 \mathrm{~mL})$ & 1.02 & 0.36 \\
SFTR-25hr (25 L) & 1.09 & 0.37 \\
Multi-Tube (100 L) & 1.06 & 0.39 \\
\hline
\end{tabular}

(b)
Figure 5. (a) Particle size distributions of different runs of $\mathrm{CaCO}_{3}$ synthesis, (b) comparison of span and $d_{\mathrm{v} 50}$ for different runs of $\mathrm{CaCO}_{3}$ synthesis.
$\mathrm{ZnO}$ results from the mixing of zinc nitrate and sodium hydroxide aqueous solutions (Eq. (7)).

$\mathrm{Zn}^{2+}{ }_{(\mathrm{aq})}+2 \mathrm{HO}_{(\mathrm{aq})}^{-} \rightarrow \mathrm{ZnO}{ }_{(\mathrm{s})}+\mathrm{H}_{2} \mathrm{O}$

The precipitation conditions were optimized in a previous study using a minibatch reactor [38]. The $0.10 \mathrm{M} \mathrm{Zn}^{2+}$ reaction solution was prepared by dissolving $\mathrm{Zn}\left(\mathrm{NO}_{3}\right)_{2} \cdot 6 \mathrm{H}_{2} \mathrm{O}$ in ultrapure water. The $0.11 \mathrm{M} \mathrm{NaOH}$ reaction solution was prepared by diluting a titrated solution of $1 \mathrm{M} \mathrm{NaOH}$ in ultrapure water. PAA $\left(M_{\mathrm{w}} 2000\right)$ at $0.05 \mathrm{wt}-\%$ was added to the $\mathrm{NaOH}$ reaction solution for a better control of the precipitated powder characteristics. After reaction, the suspended powder was washed four times with ultrapure water, before being filtered and dried for $24 \mathrm{~h}$ at $70^{\circ} \mathrm{C}$.

$\mathrm{ZnO}$ precipitation was conducted in a $400 \mathrm{~cm}^{3}$ vessel, called batch reactor $\mathrm{B}$, and in a $40 \mathrm{~cm}^{3}$ vessel, called minibatch reactor $\mathrm{MB}$, both placed in a thermostatic water bath at $90^{\circ} \mathrm{C}$. The same experiments were also performed using the SFTR, called $\mathrm{S}$ (see Tab. 2). Dodecane was used as the segmenting fluid. The reaction was carried out at $90^{\circ} \mathrm{C}$ and the residence time was 10 min. All synthesis conditions are summarized in Tab. 2 .

Table 2. Description of the process used (micromixer and reactor) for the different precipitated $\mathrm{ZnO}$ samples.

\begin{tabular}{lllll}
\hline Sample & Micromixer & Reactor & & Productivity \\
\hline B & none & Batch & $400 \mathrm{~cm}^{3}$ & $2 \mathrm{~g} / \mathrm{batch}$ \\
B-Y & Y & Batch & $400 \mathrm{~cm}^{3}$ & $2 \mathrm{~g} / \mathrm{batch}$ \\
B-V & Roughton (Vortex) & Batch & $400 \mathrm{~cm}^{3}$ & $2 \mathrm{~g} / \mathrm{batch}$ \\
MB-Y & Y & Minibatch & $40 \mathrm{~cm}^{3}$ & $0.2 \mathrm{~g} / \mathrm{batch}$ \\
MB-V & Roughton & Minibatch & $40 \mathrm{~cm}^{3}$ & $0.2 \mathrm{~g} / \mathrm{batch}$ \\
S-V & Roughton & SFTR & $0.2 \mathrm{~cm}^{3}$ & $4 \mathrm{~g} / \mathrm{h}$ \\
\hline
\end{tabular}

\subsection{Influence of the Process}

Whatever the process (reactor and micromixer), pure and crystalline $\mathrm{ZnO}$ nanoparticles were obtained by precipitating zinc nitrate and sodium hydroxide in the presence of PAA at $90^{\circ} \mathrm{C}$, as shown with the diffractogram of the sample $\mathrm{S}-\mathrm{V}$ in Fig. 6. This diffractogram matches the $\mathrm{ZnO}$ pattern of wurtzite (ICDD 075-0576). Crystallite sizes were calculated using the [100] reflection and are given in Tab. 3. All powders present nanodomains in the same range, varying between 24 and $30 \mathrm{~nm}$.

The TG curve of $\mathrm{S}-\mathrm{V}$ is presented in Fig. 7. The first weight loss between 30 and $200^{\circ} \mathrm{C}$ was attributed to the removal of physically adsorbed water. The second weight loss between 200 and $400^{\circ} \mathrm{C}$ is the consequence of two phenomena: the decomposition of chemically bound hydroxyl groups and 


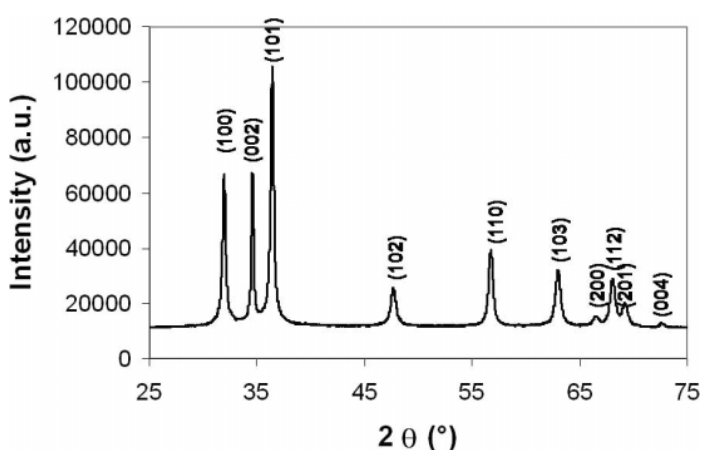

Figure 6. XRD pattern of $\mathrm{ZnO}$ synthesized with the SFTR (sample S-V).

the removal of the organic compound PAA that was adsorbed at the surface. The total weight loss measured for the different samples is in the same range, between 12.7 and $14.5 \%$, as shown in Tab. 3.

There is, however, a significant influence of both micromixer and type of reactor on the specific surface area and on the PSD. This is demonstrated by the three characteristic sizes of the PSD $\left(d_{\mathrm{v} 10}, d_{\mathrm{v} 50}, d_{\mathrm{v} 90}\right)$ given in Tab. 3 and the cumulative distributions compared in Fig. 8.

The span is calculated from Eq. (8), giving an indication of the width of the PSD:

$\operatorname{span}=\frac{d_{\mathrm{v} 90}-d_{\mathrm{v} 10}}{d_{\mathrm{v} 50}}$

If no micromixer is used (sample B), the resulting particles from the batch reactor have a span of 1.8 with sizes ranging from 100 to $700 \mathrm{~nm}$ and a specific surface area of $45 \mathrm{~m}^{2} \mathrm{~g}^{-1}$. The lack of control on the mixing and thus the initial supersaturation presumably leads to such heterogeneity in the final powder.

Using a micromixer with the batch reactor (Y- or Roughton configuration) decreases the particle size and a span below 1.0 is attained. With the Y-micromixer, the $d_{\mathrm{v} 50}$ of the particles is around $100 \mathrm{~nm}$ (B-Y: $109 \mathrm{~nm}$ ), whereas with the Roughton micromixer the $d_{\mathrm{v} 50}$ of the particles is below $100 \mathrm{~nm}$ (B-V: $82 \mathrm{~nm})$. The Roughton configuration allows a better mixing as shown by the calculation of the Kolmogorov length and the

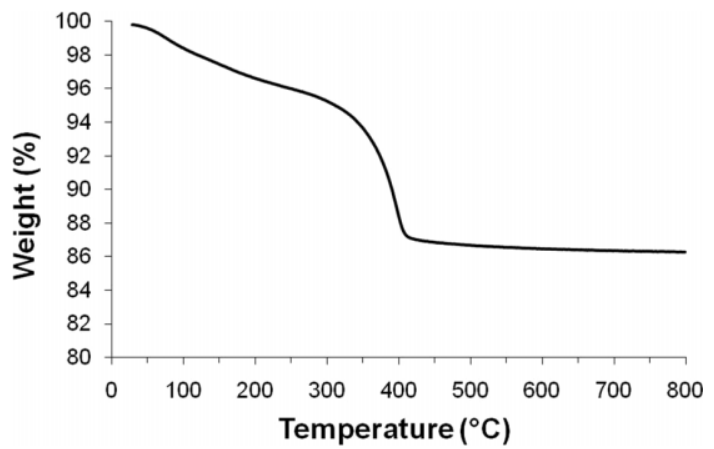

Figure 7. TG curve obtained for $\mathrm{ZnO}$ synthesized with the SFTR (sample S-V). Heating rate $10^{\circ} \mathrm{C} \mathrm{min}^{-1}$ in flowing air $\left(30 \mathrm{~mL} \mathrm{~min}^{-1}\right)$.

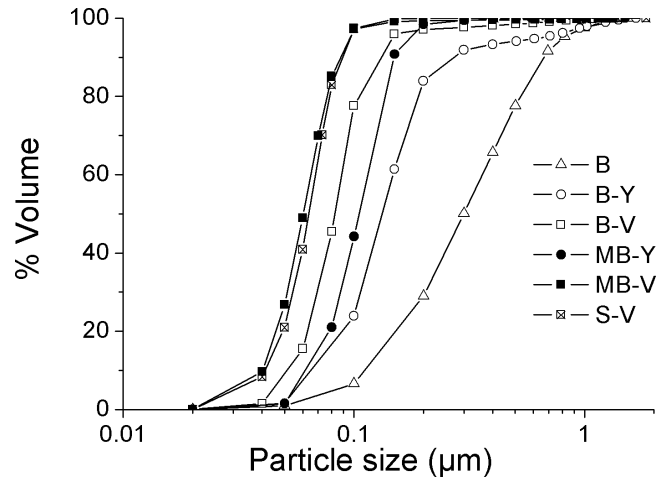

Figure 8. Particle size distributions of $\mathrm{ZnO}$ powders measured by centrifugal sedimentation (CPS).

estimated mixing time (Tab. 2: $17 \mathrm{~ms}$ with the Y-configuration and $10 \mathrm{~ms}$ with the Roughton configuration). Consequently, with a faster mixing, it can be assumed that the local supersaturation will be higher, leading to smaller particle sizes. These trends with the two mixer geometries were also observed for the minibatch and SFTR reactors.

The reactor volume also has an influence on the final PSD. By using the same micromixer (Y or Roughton), the $d_{\mathrm{v} 50}$ of the powder is higher and the specific surface area is lower in a large-volume batch reactor. The particles $\mathrm{B}-\mathrm{Y}$ and $\mathrm{B}-\mathrm{V}$ also

Table 3. Results of $S_{\mathrm{BET}}, d_{\mathrm{BET}}, d_{\mathrm{XRD}}$, particle size distribution (PSD), and weight loss measured by TCA of $\mathrm{ZnO}$ powders obtained through different processes.

\begin{tabular}{|c|c|c|c|c|c|c|c|c|}
\hline Sample & $\begin{array}{l}\left.S_{\mathrm{BET}}\right] \\
{\left[\mathrm{m}^{2} \mathrm{~g}^{-1}\right.}\end{array}$ & $\begin{array}{l}d_{\mathrm{BET}} \\
{[\mathrm{nm}]}\end{array}$ & $\begin{array}{l}d_{\mathrm{XRD}} \\
{[\mathrm{nm}]}\end{array}$ & $d_{\mathrm{v} 10}[\mathrm{~nm}]$ & $\begin{array}{c}\text { PSD } \\
d_{\mathrm{v} 50}[\mathrm{~nm}]\end{array}$ & $d_{\mathrm{v} 90}[\mathrm{~nm}]$ & $\begin{array}{l}\text { Span } \\
\left(d_{\mathrm{v} 90}-d_{\mathrm{v} 10}\right) / d_{\mathrm{v} 50}\end{array}$ & $\begin{array}{l}\text { Weight loss at } 800^{\circ} \mathrm{C} \\
{[\%]}\end{array}$ \\
\hline B & 45 & 24 & 27 & 119 & 307 & 674 & 1.8 & 13.7 \\
\hline B-Y & 57 & 19 & 30 & 67 & 109 & 178 & 1.0 & 12.7 \\
\hline B-V & 64 & 17 & 28 & 56 & 82 & 116 & 0.7 & 13.7 \\
\hline MB-Y & 75 & 14 & 24 & 65 & 104 & 147 & 0.8 & 14.5 \\
\hline MB-V & 78 & 14 & 23 & 40 & 60 & 85 & 0.7 & \\
\hline$S-V$ & 68 & 16 & 24 & 42 & 64 & 86 & 0.7 & 13.3 \\
\hline
\end{tabular}


look bigger and less homogeneous in size in the SEM pictures (Fig. 5). So, by increasing the reacting volume from only 40 to $400 \mathrm{~cm}^{3}$, the homogeneity of the powder and the mean particle size were significantly changed. This is typical of scale-up in processes, which are mainly attributed to poor homogeneity inside the reactor volume, leading to concentration variations, accumulation zones, and also to heat transfer discrepancies, which can affect the precipitation kinetics (nucleation and growth) and hence the final powder quality.

With a smaller volume of reaction a better homogeneity is ensured in the minibatch and the SFTR reactors and a narrower PSD is obtained. SEM and TEM micrographs in Fig. 9 give a qualitative view of the nanometric sizes of these particles and their narrow size distributions, confirmed with the PSD measurement by the CPS centrifugal method (Fig. 4). These results show that the particles are polycrystalline with primary particles from the XRD line broadening being around 23 to $30 \mathrm{~nm}$ and the redispersable particles being 60 to $100 \mathrm{~nm}$. Finally, one can observe in Fig. 8 that the PSDs of MB-V and $\mathrm{S}-\mathrm{V}$ are almost superimposed. This illustrates the possibility to optimize the precipitation parameters at the lab scale (a few $\mathrm{mg} / \mathrm{batch}$ ) and transfer directly these parameters to the SFTR
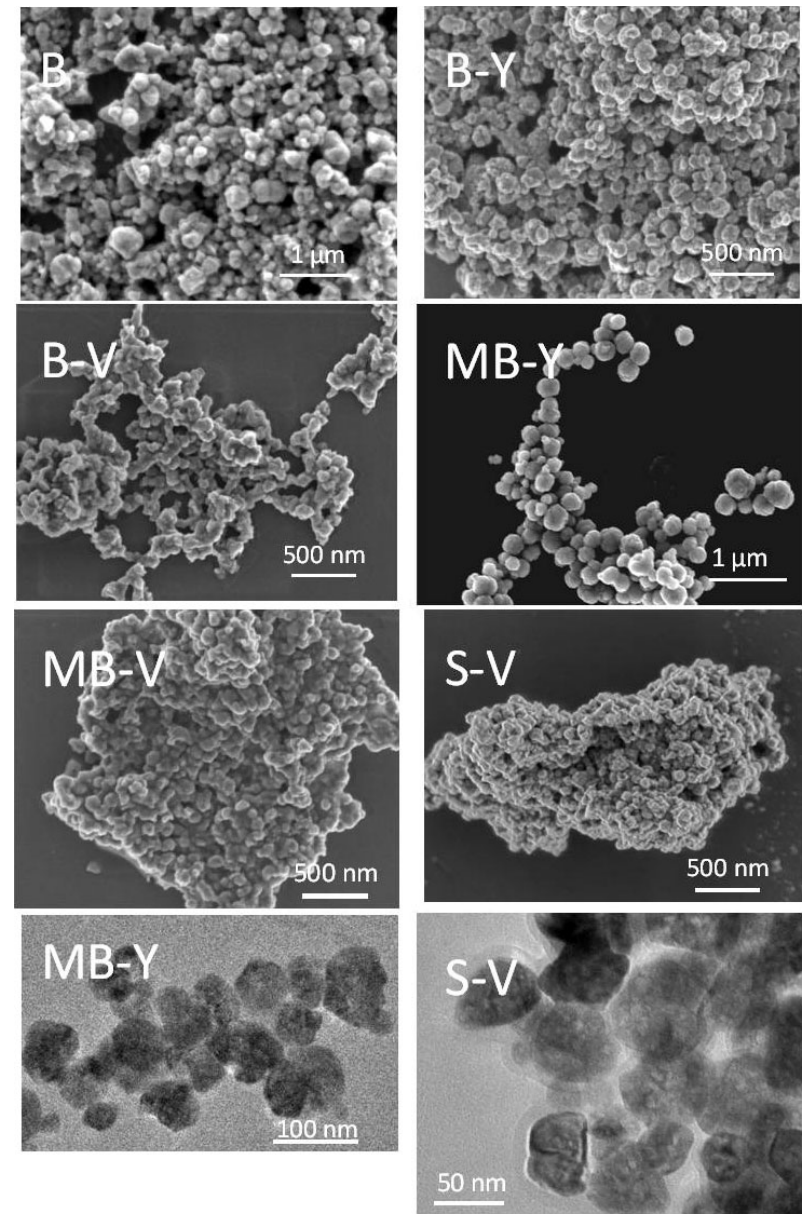

Figure 9. SEM and TEM (MB-Y and S-V) micrographs of $\mathrm{ZnO}$ powders obtained from different processes. for a pilot production scale ( $\mathrm{g}$ to $\mathrm{kg} / \mathrm{h}$, depending on the number of tubes working in parallel and the solubility of the product powder), maintaining the high-quality characteristics of the final powder.

\subsection{Robustness of the SFTR}

The SFTR offers the benefit of a continuous process rather than stop-start batch reactors. Moreover, the SFTR allows the production of higher-quality materials due to the segmenting fluid which produces homogeneous microreactors. To demonstrate that the powder quality for nanosized powders could be maintained over a realistic working time, $\mathrm{ZnO}$ precipitation was run for $12 \mathrm{~h}$. Some aliquots of the aqueous suspension were collected at different times for the measurement of the particle size distribution by CPS. The results are shown in Fig. 10. The PSD remains very narrow over the considered period of time with an excellent stability $\left(d_{\mathrm{v} 50}=64.6 \pm 2.1 \mathrm{~nm}\right)$. The particle morphology and phase purity as well as the specific surface area were also constant over the same period (not shown). The SFTR produced $50 \mathrm{~g}$ of powder with an excellent control of its properties. Such quantity is a minimum for further investigations on the ceramic processing and sintering tests, which are currently under investigation to determine their applicability for real-ceramic applications.

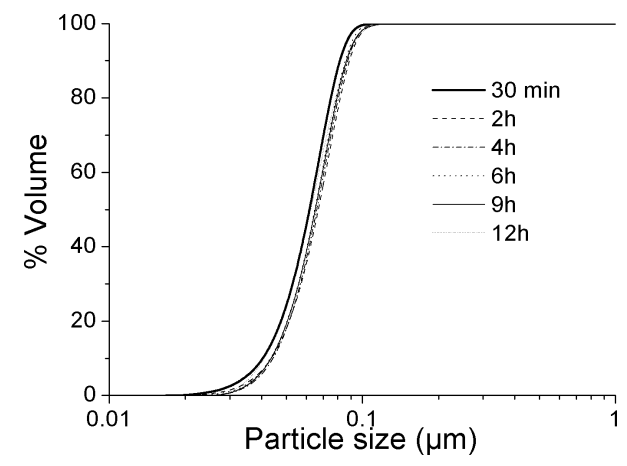

Figure 10. Particle size distributions of $\mathrm{ZnO}$ powders during $12 \mathrm{~h}$ of continuous precipitation using the SFTR, measured by centrifugal sedimentation (CPS).

It was also interesting to consider the effect of the drying step on the agglomeration of the powder, as most applications prefer the use of dried powders as raw materials. The PSD was measured on an aliquot of the aqueous phase just after collection from the reactor and on resuspended powder previously dried by two different processes: classical drying in an oven at $70{ }^{\circ} \mathrm{C}$ during $24 \mathrm{~h}$ and freeze-drying. The results are shown in Fig. 11. This figure clearly shows that while freeze-drying allows the redispersion of the powder to give the same PSD as before drying, the classical oven drying leads to the formation of a small percentage of agglomerates ( $\sim 8 \%$ of the volume). This is a very important aspect for the technological breakthrough of nanosized powders. All too often PSDS are given from TEM images with the degree of agglomeration being qualitatively assessed as low or softly agglomerated. Here, we 
properties and accompanies the development and optimization of new materials in the growing market of nanotechnologies as well as high added-value powders in general.

\section{Acknowledgements}

The authers would like to thank the Swiss cost office, COST Action 539 and the Swiss Federal Office for Education and Science (CTI-TOPNANO 21) for support.

The authors have declared no conflict of interest.

Figure 11. Particle size distributions of $\mathrm{ZnO}$ powders synthesized with the SFTR, in suspension after the reaction, after a classical drying of $24 \mathrm{~h}$ at $70^{\circ} \mathrm{C}$, and after freeze-drying, measured by centrifugal sedimentation (CPS).

demonstrate the capacity of the SFTR to produce powders which can be redispersed after the drying step, thus allowing for more economical transport.

\section{Conclusions}

Using a plug flow segmented fluid microreactor, the SFTR, successful scale-out and process intensification for ultrafine $\mathrm{CaCO}_{3}, \mathrm{BaTiO}_{3}$, and nanosized $\mathrm{ZnO}$ powders were investigated. The chemistry and operational conditions were optimized using minibatch systems $(20-100 \mathrm{~mL})$ and the powder quality could be maintained throughout the scale-up or process intensification steps. This is of key importance in moving from lab scale to pilot and/or production scale, reducing time and cost in the development and testing of new products.

The process intensification was demonstrated by producing $3-7 \mathrm{~kg}$ batches of tetragonal $\mathrm{BaTiO}_{3}$ powders $\left(d_{\mathrm{v} 50}=\right.$ $173 \pm 3 \mathrm{~nm}$ ) and with excellent batch-to-batch reproducibility over seven to ten batches. The scale-out or numbering-up capacity of the SFTR was demonstrated for a nanostructured $\mathrm{CaCO}_{3}\left(d_{\mathrm{v} 50} \approx 500 \mathrm{~nm}\right)$ by scaling-out from one to six tubular reactors run in parallel representing a scale-out/-up ratio of 5000 without loss of powder quality. The SFTR's potential for nanosized powders was then demonstrated by producing $50 \mathrm{~g}$ of pure and polycrystalline $\mathrm{ZnO}$ nanoparticles. This nanopowder $\left(d_{\mathrm{v} 50}=65 \pm 2 \mathrm{~nm}\right)$ shows a very narrow PSD due to a perfect control of the precipitation conditions by using a Roughton micromixer and the reduced reaction volume after segmentation $\left(\sim 0.2 \mathrm{~cm}^{3}\right)$. An excellent reproducibility was demonstrated and in particular the very narrow PSDs were conserved over the whole $12 \mathrm{~h}$ run. Dried powders could be perfectly redispersed when using the freeze-drying method.

The ability of the SFTR to scale out the powder production from very low optimization volumes $\left(40 \mathrm{~cm}^{3}\right)$ at the laboratory scale without changes in powder quality has been clearly demonstrated. For the different chemical systems investigated the pilot quantities of powder produced allow their assessment for ceramic or other applications. The SFTR is a powerful tool for the production of powders and nanocrystals with advanced

\section{References}

[1] National Nanotechnology Initiative. http://www.nano.gov

[2] Nanoscience and Technology: A Collection of Reviews from Nature Journals (Ed: P. Rodgers), World Scientific Publishing, Singapore 2009.

[3] V. C. Tung et al., Nat. Nanotechnol. 2009, 4, 25.

[4] A. S. Edelstein, in Encyclopedia of Materials: Science and Technology (Eds: K. H. J. Buschow et al.), Elsevier, Amsterdam 2001, 5916.

[5] D. L. Schodek, P. Ferreira, M. F. Ashby, Nanomaterials, Nanotechnologies and Design, Butterworth-Heinemann, Woburn, MA 2009.

[6] M. J. Pitkethly, Mater. Today 2004, 7, 209.

[7] J. Lemaitre, N. Jongen, R. Vacassy, P. Bowen, US Patent $6458335,2002$.

[8] J. R. Burns, C. Ramshaw, Lab Chip 2001, 1, 10.

[9] T. Henkel et al., Chem. Eng. J. 2004, 101, 439.

[10] A. Gunther, K. F. Jensen, Lab Chip 2006, 6, 1487.

[11] N. Harries, J. R. Burns, D. A. Barrow, C. Ramshaw, Int. J. Heat Mass Transfer 2003, 46, 3313.

[12] M. N. Kashid, D. W. Agar, Chem. Eng. J. 2007, 131, 1.

[13] J. Jovanović et al., Ind. Eng. Chem. Res. 2010, 49, 2681.

[14] M. N. Kashid, Y. M. Harshe, D. W. Agar, Ind. Eng. Chem. Res. 2007, 46, 8420 .

[15] S. Gulati et al., J. R. Soc. Interface 2009, 6, 1742.

[16] R. Vacassy, J. Lemaitre, H. Hofmann, J. H. Gerlings, AIChE J. 2000, 46, 1241.

[17] P. Bowen et al., Key Eng. Mater. 2002, 206-213, 21.

[18] S. Guillemet-Fritsch et al., Solid State Ionics 2004, 171, 135.

[19] M. Donnet, N. Jongen, J. Lemaître, P. Bowen, J. Mater. Sci. Lett. 2000, 19, 749.

[20] N. Jongen et al., Chem. Eng. Technol. 2003, 26, 303.

[21] E. M. Chan, A. P. Alivisatos, R. A. Mathies, J. Am. Chem. Soc. 2005, 127, 13854.

[22] D. R. Clarke, J. Am. Ceram. Soc. 1999, 82, 485.

[23] U. Ozgur et al., J. Appl. Phys. 2005, 98, 041301.

[24] V. A. Fonoberov, A. A. Balandin, J. Nanoelectron. Optoelectron. 2006, 1, 19.

[25] P. Bowen et al., Key Eng. Mater. 2002, 206-213, 21.

[26] D. L. Marchisio, F. Omegna, A. A. Barresi, P. Bowen, Ind. Eng. Chem. Res. 2008, 47, 7202.

[27] J. Chen, C. Zheng, G. A. Chen, Chem. Eng. Sci. 1996, 51, 1957. 
[28] A. J. Mahajan, D. J. Kirwan, AIChE J. 1996, 42, 1801.

[29] R. J. Demyanovich, J. R. Bourne, Ind. Eng. Chem. Res. 1989, 28,825 .

[30] J. Garside, O. Sohnel, Precipitation: Basic Principles and Industrial Applications, Butterworth-Heinemann, Woburn, MA 1993.

[31] R. J. Demyanovich, J. R. Bourne, Ind. Eng. Chem. Res. 1989, 28,830 .

[32] P. Bowen, J. Dispers. Sci. Technol. 2002, 23, 631.

[33] A. Testino et al., Chem. Mater. 2005, 17, 5346.
[34] M. Donnet et al., Langmuir 2005, 21, 100.

[35] U. Aschauer, J. Ebert, A. Aimable, P. Bowen, Cryst. Growth Des. 2010, 10, 3956.

[36] U. Aschauer, D. Spagnoli, P. Bowen, S. C. Parker, J. Colloid Interface Sci. 2010, 346, 226.

[37] M. Donnet, A. Aimable, J. Lemaître, P. Bowen, J. Phys. Chem. B 2010, 114, 12058.

[38] A. Aimable, M. T. Buscaglia, V. Buscaglia, P. Bowen, J. Eur. Ceram. Soc. 2010, 30, 591. 